\title{
Metagenomics of rumen bacteriophage from thirteen lactating dairy cattle
}

Elizabeth M Ross ${ }^{1,2,3^{*}}$, Steve Petrovski ${ }^{1,2}$, Peter J Moate ${ }^{1}$ and Ben J Hayes ${ }^{1,2,3}$

\begin{abstract}
Background: The bovine rumen hosts a diverse and complex community of Eukarya, Bacteria, Archea and viruses (including bacteriophage). The rumen viral population (the rumen virome) has received little attention compared to the rumen microbial population (the rumen microbiome). We used massively parallel sequencing of virus like particles to investigate the diversity of the rumen virome in thirteen lactating Australian Holstein dairy cattle all housed in the same location, 12 of which were sampled on the same day.

Results: Fourteen putative viral sequence fragments over $30 \mathrm{Kbp}$ in length were assembled and annotated. Many of the putative genes in the assembled contigs showed no homology to previously annotated genes, highlighting the large amount of work still required to fully annotate the functions encoded in viral genomes. The abundance of the contig sequences varied widely between animals, even though the cattle were of the same age, stage of lactation and fed the same diets. Additionally the twelve animals which were co-habited shared a number of their dominant viral contigs. We compared the functional characteristics of our bovine viromes with that of other viromes, as well as rumen microbiomes. At the functional level, we found strong similarities between all of the viral samples, which were highly distinct from the rumen microbiome samples.

Conclusions: Our findings suggest a large amount of between animal variation in the bovine rumen virome and that co-habiting animals may have more similar viromes than non co-habited animals. We report the deepest sequencing to date of the rumen virome. This work highlights the enormous amount of novelty and variation present in the rumen virome.
\end{abstract}

Keywords: Virome, Rumen, Bacteriophage, Metagenomics

\section{Background}

The value of domestic ruminants (cattle, sheep and goats) comes from their ability to convert, by means of rumen fermentation, low-quality forages into high quality, high protein products (milk and meat) suitable for human consumption [1]. The rumen, the first section of the ruminant stomach, contains symbiotic microorganisms that breakdown ingested food. These microorganisms include Eukarya, Bacteria, Archaea [2], and large numbers $\left(>10^{7} \mathrm{pfu} \mathrm{ml}^{-1}\right)$ of virus including bacteriophage [3]. The entire bacteriophage or 'phage' population in a sample is termed a virome.

\footnotetext{
* Correspondence: elizabeth.ross@depi.vic.gov.au

'Department of Environment and Primary Industries, Bundoora, VIC 3086, Australia

${ }^{2}$ Dairy Futures Cooperative Research Centre, Bundoora, VIC 3086, Australia Full list of author information is available at the end of the article
}

Massively parallel sequencing (MPS) is a technology which allows the generation of several million DNA sequences in parallel. MPS has been used to investigate the Bacterial and Archaeal populations of the bovine (Bos taurus) rumen [4-7]. However, MPS studies on the rumen virome are scant. One study [8] sequenced 425 Mbp of viral DNA from the rumen of three animals, one lactating, one non lactating and one culled animal. The MPS study [8] and earlier studies such as [9], both suggest that there are large amounts of variation in the bovine rumen virome. However some of the variation may have been due to external sources (diet, lactation state, location, time of sampling).

Since the recent rumen virome study [8], there have been technological advances that have allowed the volume of sequence data to increase by an order of magnitude. These advances now enable the sequencing of viromes from a greater numbers of animals, resulting in

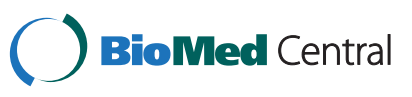


the ability to gain an insight into natural variation between animals. MPS virome studies have made it possible to study viral genomes even when the host bacterium cannot be cultured. However, there are significant challenges involving analysis of MPS virome data [10]. Tools for the analysis of virome samples [11-13] are becoming more readily available allowing estimates of virome characteristics such as species richness. The combination of published analysis techniques, along with the increased ability to generate data has allowed the field to switch from descriptive analyses to quantitative investigations of how various factors (such as host effects or temporal variation) affect the virome [14].

To access the amount of variation in the rumen virome we extracted virus like DNA from the rumen fluid of 13 lactating dairy cattle. Twelve of the 13 cows were housed together, fed the same diet, had a shared location and were sampled on the same day, allowing us to investigate the magnitude of natural variation in the rumen virome.

\section{Results and discussion Assembly and annotation}

We sequenced virus like particles from the rumen fluid of 13 lactating Australian Holstein dairy cattle on the HiSeq2000. Each library contained between 2 and 57 million read pairs (Table 1). After a combined assembly of all samples, 14 contigs were greater than $30 \mathrm{Kbp}$ in length. These contigs were manually inspected for even coverage and annotated (Figure 1). Three of the contigs have all the genes necessary for a complete functional phage genome (contigs $\mathrm{A}, \mathrm{H}$ and $\mathrm{K}$ ). We mapped our sequence reads back to the complete set of contigs. While up to $57.5 \%$ of reads mapped to the whole assembly, only $0.84 \%$ of reads mapped to contigs greater than $10 \mathrm{Kbp}$ (Figure 2a). This would suggest that although we have assembled up to $57 \%$ of the bovine rumen virome, most of what has been assembled is still highly fragmented, and contained in small contigs.

Five of the 14 largest contigs were observed in all samples ( $\geq 10$ reads mapping back to contig), with all 14 observed in the sample from animal 2202 (which had the deepest sequence coverage; (Table 1). Despite over a third of the most common contigs being represented in all samples, the most abundant contig only accounted for $0.24 \%$ of sequence reads (contig $\mathrm{H}$ in animal 2165; Figure $2 \mathrm{~b}$ ). There was a strong correlation between the sequencing depth of the sample and the percentage of reads that mapped back to the assembly (contigs > $5 \mathrm{Kbp}$; $r=0.952$ ), partly reflecting the fact that the contigs were assembled from the same data. As sequence depth has a strong impact on contig size, it is likely that we have only assembled genomes from the most dominant phage species in the bovine rumen.
Of the 815 putative genes in the 14 largest contigs, $61.5 \%$ were assigned an unknown function because all of the significant BLAST hits were themselves unannotated. Genes involved with either DNA replication or repair consisted $13.3 \%$ of putative genes, $9.2 \%$ of putative genes were structural and $1.8 \%$ appear to be involved in lysis. Completely novel (no significant BLAST hits to the public nt database) putative genes made up an additional $13.0 \%$. These novel and unannotated genes may encode unknown functions including those involved in cell lysis or lytogenic reproduction of the phage.

Phage may be integrated into the genomes of their host prokraryotes. To look for evidence of this we aligned sequence data from the microbiomes of the same samples from [15] to the 14 largest contigs. All of the largest contigs were observed in one or more microbiome samples. This could be explained by viral contamination of the microbiome. Such contamination would result in the same proportions of viral contigs in the microbiome as are observed in the virome. Therefore the proportions of reads that map to the viral contigs in the microbiome were compared to the virome. The abundance of two contigs, $\mathrm{E}$ and $\mathrm{M}$, were significantly higher in the microbiome than expected under the viral contamination model ( $\mathrm{p}<0.05$ after a Bonferroni correction). This is evidence that these two phage are integrating into the prokaryote genomes. However this hypothesis that cannot be validated until the host genomes are sequenced or the host and phage and isolated and cultured. Additionally, contigs A, B, C, E, $\mathrm{F}, \mathrm{G}$ and $\mathrm{N}$ show homology to genes involved in lysis, suggesting that they may be lytic, however this is again speculation until the phage are isolated.

\section{Variation in contig abundance between animals}

The twelve co-habited animals' viromes appear to be dominated by contigs D, E and J, while animal 2202's virome shows a much more even coverage between contigs (Figure 2b). However these contigs only account of $<0.34 \%$ of the viromes of the co-habited animals. There also appears to be a heterogeneity in the distributions of contigs between animals (Figure 2b-c). Contig $\mathrm{H}$ has a high abundance in cow 2165 (10,480 reads mapped back to the contig), but is almost below detectable limits in the deepest sequenced animal (2202, only 10 reads mapped back to the contig) and is undetectable in cow 657 (0 reads mapped back to the contig). The evenness observed in the virome of animal 2202 possibly hints that the rumen viral community is not dominated by a single species, but has a large number of taxa with a reasonably even distribution. This evenness of distribution was likely not observed in our other samples due to their limited sequence depth resulting in many unassembled species. To assess the distribution on an independent set 
Table 1 Virome sequence volume, alignments and richness

Alignments to other viromes (\%)

Richness by CatchAll

\begin{tabular}{|c|c|c|c|c|c|c|c|c|c|c|c|}
\hline \multirow[b]{2}{*}{ Sample } & \multirow[b]{2}{*}{$\begin{array}{l}\text { Data trimmed } \\
\text { (Read pairs) }\end{array}$} & & \\
\hline & & $\begin{array}{l}\text { Cow } \\
6993[8]\end{array}$ & $\begin{array}{c}\text { Cow } \\
7664[8]\end{array}$ & $\begin{array}{c}\text { Cow } \\
7887[8]\end{array}$ & $\begin{array}{c}\text { Swine } \\
\text { faeces }[18]^{+}\end{array}$ & $\begin{array}{c}\text { Human } \\
\text { saliva }[19]^{+}\end{array}$ & Pond $[20]^{+}$ & Best model $^{B}$ & $\begin{array}{l}\text { Estimated total } \\
\text { species } \pm \text { S.E. }\end{array}$ & $\begin{array}{c}\text { Best discounted } \\
\text { model }^{\mathrm{D}}\end{array}$ & $\begin{array}{l}\text { Estimated total } \\
\text { species } \pm \text { S.E. }\end{array}$ \\
\hline Cow $2202^{C}$ & $52,239,452$ & 3.22 & 3.25 & 1.86 & 0.07 & $<0.01$ & $<0.01$ & 2MixedExp & $5550786 \pm 465864.1$ & SingleExp & $18412.6 \pm 1544.2$ \\
\hline Cow 657 & $2,116,650$ & 4.86 & 4.91 & 3.12 & 0.05 & $<0.01$ & $<0.01$ & 3MixedExp & $4679342 \pm 676180.6$ & 2MixedExp & $26795.8 \pm 3871.9$ \\
\hline Cow 1995 & $3,410,438$ & 3.88 & 4.69 & 2.69 & 0.04 & $<0.01$ & $<0.01$ & 2MixedExp & $5952188.7 \pm 295239.5$ & SingleExp & $5313.4 \pm 263.5$ \\
\hline Cow 2028 & $6,956,332$ & 3.57 & 4.24 & 2.30 & 0.04 & $<0.01$ & $<0.01$ & 3MixedExp & $6515260.9 \pm 338382$ & 2MixedExp & $3369.8 \pm 175$ \\
\hline Cow 2042 & $4,048,214$ & 3.19 & 3.71 & 2.22 & 0.03 & $<0.01$ & $<0.01$ & 2MixedExp & $7904757.2 \pm 622624.3$ & SingleExp & $9023.1 \pm 707.6$ \\
\hline Cow 2165 & $4,362,890$ & 4.57 & 5.14 & 3.20 & 0.04 & $<0.01$ & $<0.01$ & 4MixedExp & $10353681.8 \pm 119769.7$ & 3MixedExp & $1376492.9 \pm 15923$ \\
\hline Cow 3060 & $3,899,392$ & 4.53 & 5.01 & 3.35 & 0.05 & $<0.01$ & $<0.01$ & 2MixedExp & $5805999.9 \pm 388938$ & SingleExp & $11428.7 \pm 765.5$ \\
\hline Cow 4570 & $4,365,420$ & 3.41 & 4.05 & 2.53 & 0.04 & $<0.01$ & $<0.01$ & 4MixedExp & $22410379.6 \pm 511083.2$ & 3MixedExp & $4126756.3 \pm 94113.3$ \\
\hline Cow 5679 & $3,681,922$ & 3.70 & 4.84 & 2.67 & 0.04 & $<0.01$ & $<0.01$ & 3MixedExp & $6098575.2 \pm 404293.2$ & 2MixedExp & $6772.1 \pm 448.5$ \\
\hline Cow 6833 & $3,455,882$ & 3.58 & 4.18 & 2.55 & 0.05 & $<0.01$ & $<0.01$ & 3MixedExp & $7427673 \pm 2447886.4$ & 2MixedExp & $30174.7 \pm 9943.8$ \\
\hline Cow 6857 & $8,998,900$ & 4.18 & 4.71 & 2.77 & 0.04 & $<0.01$ & $<0.01$ & 3MixedExp & $7916340 \pm 2025360.6$ & 2MixedExp & $23466.2 \pm 6003$ \\
\hline Cow 6870 & $3,906,564$ & 4.49 & 5.88 & 3.60 & 0.03 & $<0.01$ & $<0.01$ & 2MixedExp & $4794540.3 \pm 304303.4$ & SingleExp & $13438.6 \pm 852.7$ \\
\hline Cow 7939 & $5,119,322$ & 3.45 & 5.02 & 2.84 & 0.03 & $<0.01$ & $<0.01$ & 2MixedExp & $6757358.4 \pm 455261$ & SingleExp & $7504.3 \pm 495.6$ \\
\hline Average* & $8,197,029$ & 3.89 & 4.59 & 2.75 & 0.04 & $<0.01$ & $<0.01$ & - & $7,858,991$ & - & 435,304 \\
\hline
\end{tabular}

${ }^{*}$ Average of all samples from this study.

'Cannulated animal.

Amount of sequence remaining after removal of poor quality bases.

Multiple samples from study combined.

${ }^{B}$ Best Model as assessed by the CatchALL program.

Best Model that has been corrected using the assumption of overrepresented singletons. 


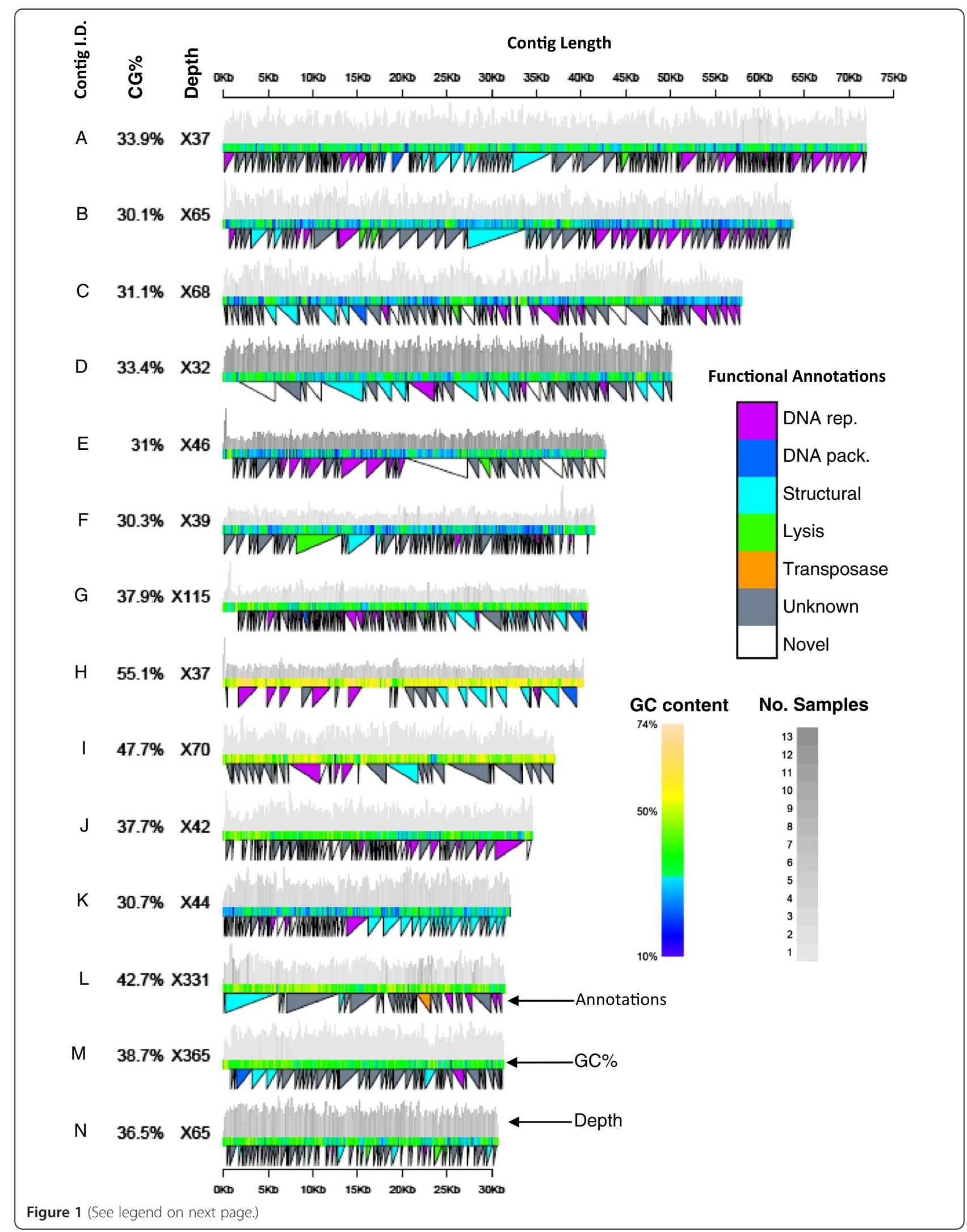


(See figure on previous page.)

Figure 1 Virome contigs greater than $\mathbf{3 0} \mathbf{~ k b p}$. The median coverage of the contig and the GC\% are shown to the left of each contig. The colour of the centre bar represents the GC\% in a 100 bp sliding window. The grey lines on top of each contig represent the sequence depth, as determined by aligning reads to the contig using BWA. The shade of grey represents the number of different samples that have reads mapping to that position. The triangles below the contig show the function of the annotation at that position. DNA rep. = DNA replication and repair, DNA pack. = DNA packaging, Unknown = all significant BLAST hits were to unannotated subjects, Novel = no significant BLAST hit.

of contigs we aligned our reads to contigs assembled from the independent set of animals from [8]. Between $0.22 \%$ and $0.70 \%$ of our reads aligned to the contigs. The distribution of contigs was much more even when these independent contigs were used (Figure 2d). A principle component analysis did not separate the independently housed animal from the co-house animal, although as less than $1 \%$ of the reads from any virome aligned, this should be interpreted with caution. The evenness of the phage population concurs with the findings of [8] that even the most abundant phage species represent only a small proportion of the rumen virome. Our finding that the most common of phage contigs only represent $0.24 \%$ of the virome is consistent with this. The higher degree of similarity between the viromes of the 12 co-habited animals and animal 2202 may suggest that there is transfer of viral species between the hosts within a herd, although 2202 was also cannulated, this confounding effect is not possible to resolve without further virome data. Still the variation between co-habited animals (for example contig $\mathrm{H}$ in animals 2165 and 657) illustrates that any transfer between animals does not maintain a completely homogeneous herd. Whether the within herd variation is related to traits that are related to rumen microbiome functions, such as methane emission levels or bloat susceptibility is yet to be seen, however this dataset shows that there is natural within herd virome variation which should be investigated. Future studies may explore the relationship between this variation and host traits such as disease susceptibility and methane emissions.

\section{Species richness}

Based on our sequence data, it is clear that estimations of rumen phage diversity using electron microscopy of less than 40 species per host summerised in [16] are dramatic underestimations, and the true number of species likely lies closer to the estimate in [8] of between 4 and 27 thousand. To estimate the number of species present in each of our samples, we used CatchAll [13]. The estimated total number of species were between 4.6 and 22.4 million per sample (Table 1). Over estimations of richness can be caused by spurious singletons, and the biological and technical causes for these have been outlined in [13]. To account for spurious singletons we also used a model which corrects for such artefacts. This model reported between 3,370 and 4,126,756 (Table 1) species per sample. The divergence between these results and those reported in [8] is not surprising given that the authors of CatchAll consistently report richness higher than PHACCS [12], and claim that PHACCS underestimates richness. Our assembly contains $272 \mathrm{Mbp}$ of assembled sequence, if we assume an average phage genome size of $30 \mathrm{Kbp}$ this results in approximately 9073 genomes (assuming little overlap between contigs) worth of sequence in our assembly. Given that we have only assembled half of the sequence in our samples, and the assembled half would be the less diverse than the unassembled half, approximately 18 thousand genomes could be expected as a lower bound for number of species estimations. This rough calculation concurs with the CatchAll discounted model estimate for cow 2202, and together they tend to suggest that previous assessments of rumen phage richness are indeed underestimations. In any case, it is clear that there is a large amount of variation in the species that make up the $B$. taurus rumen virome. Also the large range of estimates, even under the reduced model, suggests that the viromes of some animals are much more species rich than others. This could be an indication of a more diverse prokaryote population in some animals than others. This large range should be considered in future experimental designs, as deeper sequencing of particularly diverse samples will be needed to capture a snapshot of the viral community.

\section{Comparison to other viromes}

There are a number of host associated and non host associated viromes sequenced to date. To compare these virome sequence profiles to ours we obtained reads from publically available datasets derived from published studies. Here we have only isolated viral DNA (not RNA), therefore we were unable to compare our results to studies such as that by [17], which isolated RNA viruses. We selected four different types of viromes for comparison with our data. The viromes included samples from cattle rumen, human saliva, swine faeces and non host-associated environments [8,18-20]. The reads from the published viromes were aligned to our contigs. The other bovine rumen viromes had only a small percentage (between $0.70 \%$ and $1.56 \%$; Table 2) of reads that mapped to our contigs. When we compared our reads to reads in the virome obtained from the rumen fluid of cattle as previously reported [8] only $2-5 \%$ of our reads mapped (Table 1 ). This would 

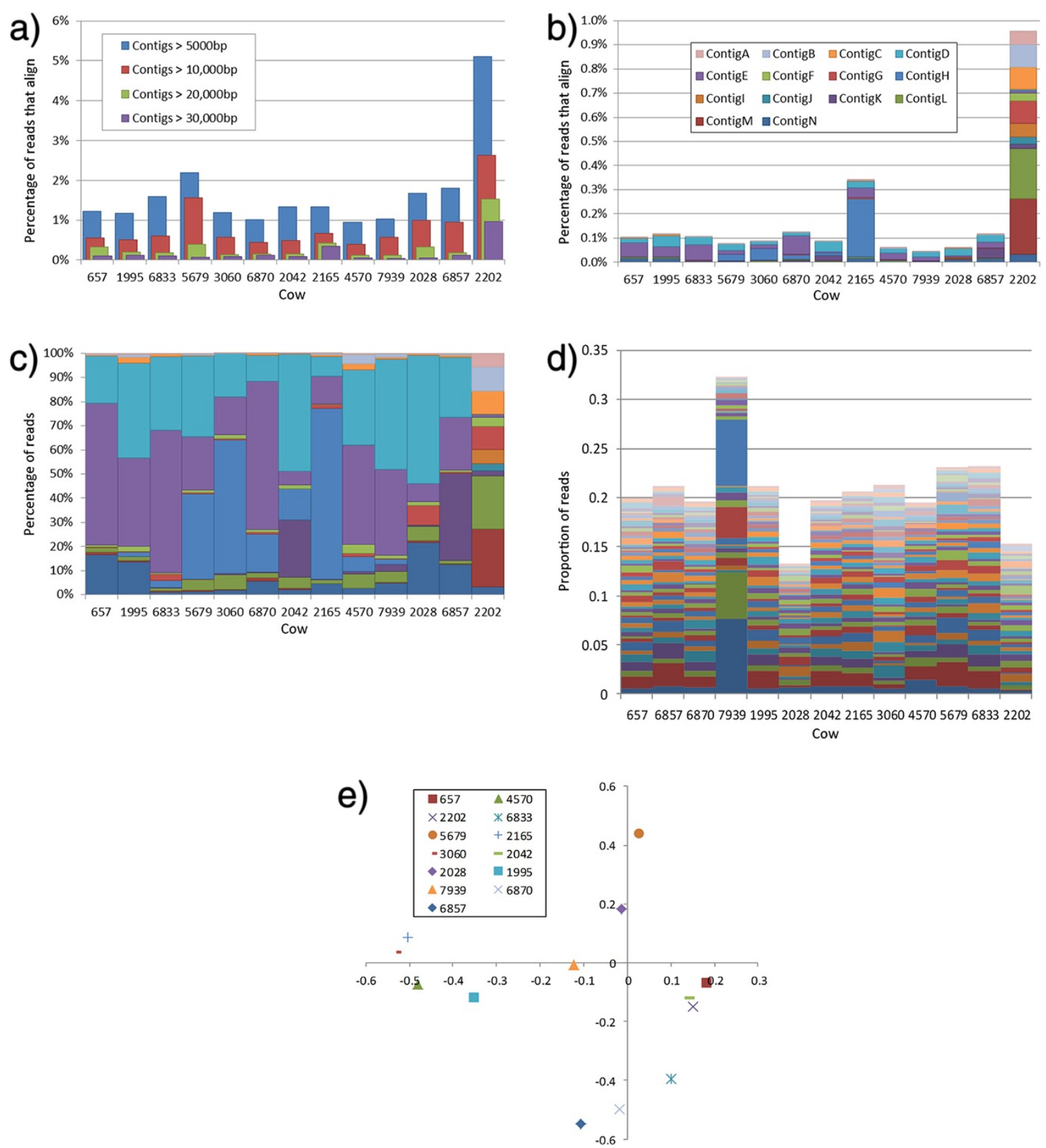

Figure 2 Between animal variation in alignments to contigs. a) Percentage of reads that map back to assembled contigs of different sizes. b) Distribution of reads that map to the 14 contigs annotated in Figure 1. c) Distribution of reads that map to the 14 contigs annotated in Figure 1 normalised to a total of $100 \%$. d) Distribution of reads that map to the 100 most abundant (in our dataset) contigs assembled from [8]. e) Principle component analysis of reads that map to the 50 most abundant contigs assembled from [8]. PC1 (X axis) explains $15.42 \%$ of the variation, PC2 (Y axis) explains $12.73 \%$ of the variation.

suggest that there are substantial differences between the viromes observed by [8] and ourselves. These differences could be a result of the phage isolation technique [21], or possibly the sequencing technology used. It is also possible that the differences were due to real variations, as there was large amounts of variation observed within our samples and it could be expected that cows from other locations and on different diets might be even more distinct.

We then compared our reads to the non-bovine viromes. The swine faeces virome [18], the human saliva virome [19] and aquaculture pond derived virome [20] did not have any reads mapping to our contigs (Table 2). We then attempted to map our reads to the 454 reads 
Table 2 Virome alignments to assembled contigs

\begin{tabular}{llllll}
\hline Sample & All contigs & Contigs $>\mathbf{5 0 0 0}$ bp & Contigs $>\mathbf{1 0 , 0 0 0}$ bp & Contigs $>\mathbf{2 0 , 0 0 0}$ bp & Contigs $>\mathbf{3 0 , 0 0 0}$ bp \\
\hline Average Rumen Virome* & $37.1 \%$ & $1.66 \%$ & $0.84 \%$ & $0.33 \%$ & $0.17 \%$ \\
Cow 6993 [8] & $0.70 \%$ & $0.03 \%$ & $0.01 \%$ & $<0.01 \%$ & $0.00 \%$ \\
Cow 766 [8] & $0.56 \%$ & $0.03 \%$ & $0.01 \%$ & $<0.01 \%$ & $<0.01 \%$ \\
Cow 7887 [8] & $1.56 \%$ & $0.03 \%$ & $<0.01 \%$ & $0.00 \%$ & $0.00 \%$ \\
Swine Faeces [18] & $0.01 \%$ & $<0.01 \%$ & $<0.01 \%$ & $0.00 \%$ & $0.00 \%$ \\
Human Saliva [19] $^{+}$ & $0.00 \%$ & $0.00 \%$ & $0.00 \%$ & $0.00 \%$ & $0.00 \%$ \\
Pond [20] $^{+}$ & $<0.01 \%$ & $0.00 \%$ & $0.00 \%$ & $0.00 \%$ & $0.00 \%$ \\
\hline
\end{tabular}

${ }^{+}$Multiple samples from study combined.

*Average of all samples from this study.

of the above mentioned databases. Less than $0.1 \%$ of our reads mapped to the swine faeces database, while less than $0.01 \%$ of our reads mapped to the human saliva or pond viromes (Table 1). There is an observable cline in the amount of our reads that map to these databases as they become less similar to our sample type, from highest from our own assembly, followed by the same sample type (bovine rumen), then an alternative species digestive tract (swine faeces), then non gut derived viromes.

This cline in reads that align to contigs from different habitats may be a result of the similar prokaryote populations within gut samples. Both swine faeces and bovine rumen are dominated by Firmicutes and Bacteroidetes phyla [6,22], while the salivary microbiome is dominated by Proteobacteria [23]. Hence the viral species in gut samples likely prey mostly on Firmicutes and Bacteroidetes, which would result in more similarities between the gut derived viromes then between the rumen and non-gut derived viromes.

\section{Functional characteristics of the rumen virome}

We also investigated the hypothesis that the functional characteristics of the bovine rumen virome were conserved between animals despite the large amount of sequence variation observed. We assigned out reads to KEGG second level pathways. Of the assigned second level KEGG pathways, Nucleotide Metabolism and Replication and Repair combined made up 57\% of virome assignments. This is in contrast to the prokaryote metagenomes of rumen fluid from animals from the same location [6] (Figure 3a). Evenness of second level KEGG pathways was significantly lower in the phage sequences compared to the microbe data (t-test, $\mathrm{p}<0.001$; Table 3 ). This unevenness in the phage dataset is likely due to the lack of genes coding for functions such as carbohydrate metabolism, which the phage relies on the host cell to provide. We then compared the functional characteristics of our sequences to other viromes. A principle component analysis revealed that all viromes clustered, and all microbial metagenomes clustered based on assignments to KEGG second level pathways (Figure 3b). These analyses reveal how the virome of a sample is functionally distinct from the microbial metagenome, and that the functional characteristics of viromes are more similar to each other than to microbial metagenomes, even when the viromes are taken from different sample types (e.g. aquaculture ponds versus rumen fluid). This is likely a reflection of the limited number of annotatable functions encoded for in the phage metagenome. Interestingly the functions coded for in our viromes are not as variable as that observed in human faeces [14]. Some of the human faeces variation may be caused by the small number of reads which could be used in the KEGG analysis due to the limited percentage of alignments and the starting data volume.

The relatively narrow functional characteristics observed in the rumen fluid virome may be in part due to the lack of homology to annotated genes in the databases used for annotation (Figure 1). The potential of novel bacteriophage derived genes to control the bacterial populations of the host will likely be of interest in both a biomedical sense (for diseases which are associated with bacterial populations such as inflammatory bowel disease) and, in the case of livestock, reducing enteric methane emissions from the rumen.

\section{Rumen virome importance}

Domestic ruminants are important production animals to people worldwide. Their ability to use microbial fermentation to provide energy for food (meat and milk), fibre (wool) and power (bullocks used as draught animals) has seen their numbers soar to 3.6 billion [24]. Because of this, researchers have long been interested in the rumen microbiome, however the virome has been neglected due to the lack of appropriate marker genes and analysis benchmarks. It is imperative that the virome is studied along with the microbiome and the host animal. The virome, being pathogens of the microbiome, can substantially alter rumen function, which in turn may hinder the ability of the host to utilise feed efficiently. 


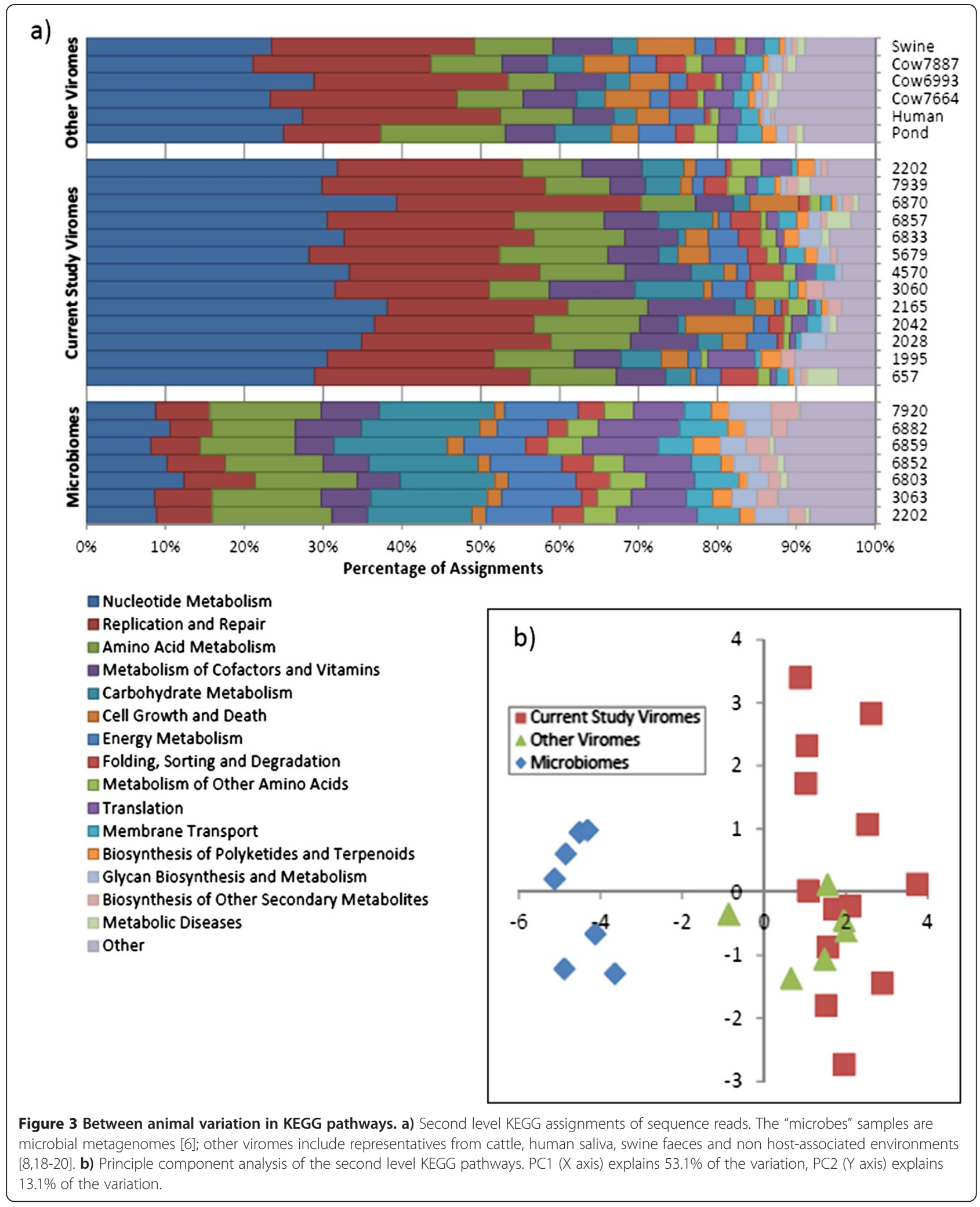

Additionally, as the microbiome in ruminants is much more closely linked to major nutrient acquisition than in humans and mice, a manipulation of the virome (and therefore the microbiome) may enable a more substantial manipulation of nutrient acquisition than is possible in monogastric animals. 
Table 3 Diversity and evenness of KEGG pathways

\begin{tabular}{|c|c|c|c|c|c|}
\hline Metagenome source & Sample & Metagenome type & Categories observed $^{\mathrm{N}}$ & Shannon diversity Index $\left(\mathrm{H}^{\prime}\right)$ & Evenness (E) \\
\hline \multirow[t]{14}{*}{ This study } & Cow $2202^{C}$ & Virome & 20 & 2.192 & 0.732 \\
\hline & Cow 657 & Virome & 21 & 2.162 & 0.710 \\
\hline & Cow 1995 & Virome & 22 & 2.299 & 0.744 \\
\hline & Cow 2028 & Virome & 20 & 2.052 & 0.685 \\
\hline & Cow 2042 & Virome & 17 & 2.011 & 0.710 \\
\hline & Cow 2165 & Virome & 16 & 1.911 & 0.689 \\
\hline & Cow 3060 & Virome & 16 & 2.150 & 0.775 \\
\hline & Cow 4570 & Virome & 15 & 2.023 & 0.747 \\
\hline & Cow 5679 & Virome & 18 & 2.184 & 0.756 \\
\hline & Cow 6833 & Virome & 19 & 2.125 & 0.722 \\
\hline & Cow 6857 & Virome & 18 & 2.142 & 0.741 \\
\hline & Cow 6870 & Virome & 16 & 1.756 & 0.633 \\
\hline & Cow 7939 & Virome & 21 & 2.200 & 0.722 \\
\hline & Average* & Virome & 18.4 & 2.093 & 0.720 \\
\hline \multirow[t]{13}{*}{ Other Studies } & Cow 6993 [8] & Virome & 36 & 2.425 & 0.677 \\
\hline & Cow $766[8]$ & Virome & 34 & 2.553 & 0.724 \\
\hline & Cow 7887 [8] & Virome & 35 & 2.580 & 0.726 \\
\hline & Swine Faeces $[18]^{+}$ & Virome & 33 & 2.398 & 0.686 \\
\hline & Human Saliva [19] ${ }^{+}$ & Virome & 36 & 2.221 & 0.620 \\
\hline & Pond $[20]^{+}$ & Virome & 31 & 2.537 & 0.739 \\
\hline & Cow $2202^{C}[6]$ & Microbial & 27 & 2.731 & 0.829 \\
\hline & Cow $3063^{C}[6]$ & Microbial & 30 & 2.794 & 0.822 \\
\hline & Cow $6803^{C}[6]$ & Microbial & 30 & 2.778 & 0.817 \\
\hline & Cow $6852^{C}[6]$ & Microbial & 29 & 2.809 & 0.834 \\
\hline & Cow $6859^{C}[6]$ & Microbial & 29 & 2.860 & 0.849 \\
\hline & Cow $6882^{C}[6]$ & Microbial & 24 & 2.752 & 0.866 \\
\hline & Cow $7920^{C}[6]$ & Microbial & 28 & 2.762 & 0.829 \\
\hline
\end{tabular}

*Average of all samples from this study.

${ }^{C}$ Cannulated animal.

${ }^{N}$ Number of second level KEGG categories observed in sample's data.

$\mathrm{H}^{\prime}$ - Shannon diversity Index.

E - Evenness $\left(\mathrm{H}^{\prime} / \mathrm{H}_{\text {max }}\right)$.

${ }^{+}$Multiple samples from study combined.

Phages have been suggested as a possible methane mitigation strategy through "bacteriophage therapy" [25]. This strategy requires identification of phage species which target the dominant methane producing Archaea in the rumen. Such approaches will benefit from metagenomic virome sequencing by the availability of additional information regarding the dominant phage species in the rumen virome, and will help establish an understanding of the stability or variability of the rumen virome over time, which may affect such therapies. Furthermore, comparison of the virome and microbiome of each rumen sample may shed light on the ecological relationship between phage and bacterium. For now, this work has illustrated the vast amount of species richness and between animal variation in the rumen virome.
Identifying which of these sequences may belong to Archaea pathogens is a significant challenge for future work.

Studies in other species have found that phages can have a protective role for their host [26], and studies investigating control of bacterial gut populations with phages have been successful [27]. The rumen virome of cattle may be the key to unlocking the secretes of the optimally functioning rumen, and therefore, should be a consideration in the quest to develop the ultimate production animal.

\section{Conclusions}

We have completed the deepest sequencing of the rumen virome to date. The results indicate large taxonomic diversity between the rumen viromes of Australian Holstein dairy 
cattle, and that animals housed together may have more similar viromes than those housed separately. However, this taxonomic variation is not reflected in the functional characteristics of the rumen virome. In fact, the rumen virome appears to be functionally conserved between animals. This suggests that while rumen viral genome sequences diverge rapidly between hosts, functional characteristics are under significant evolutionary constraint.

The observed differences between the rumen virome and the rumen microbiome likely reflect the much narrower set of functions typically encoded in a viral genome compared to a microbial genome. As MPS becomes more widely used to investigate viromes, it will be interesting to observe how these species correlate with changes in the bacterial population, or even if population differences are associated or predictive of key traits such as feed conversion efficiency and methane yield.

\section{Methods}

Animals

Animals used in this study have been previously described [6,28]. All animals were adult lactating Holstein dairy cattle. Twelve of the 13 cows were house together, fed the same diet, had a shared location and were sampled on the same day, the remaining cow (2202) was a cannulated individual which had been five months earlier. Rumen fluid from animal 2202 was removed via the fistula, rumen fluid from all other animal was collected via stomach pump. All work involving animals was approved by the Department of Environment and Primary Industries Animal Ethics Committee.

\section{Molecular techniques \& sequence quality control}

A cell pellet was removed from the rumen fluid via centrifugation and then viral DNA was extracted from the supernatant as per [29]. The putative phage DNA was sequenced on the Illumina HiSeq2000 on a 101 bp paired end run.

Poor quality sequence was removed from the dataset using dynamic trimming such that no read had an average Phred quality score below 20, and no read contained more than three bases with a Phred quality score below 15 .

\section{Assembly and annotation}

Sequence from all animals was computationally pooled and assembled into contigs using Gossamer [30]. Because of the complexity of metagenome samples, several assemblies were performed at different K-mer lengths. These assemblies were then further combined in a single Gossamer assembly $(\mathrm{K}-\mathrm{mer}=51)$. The 14 contigs that were greater than $30 \mathrm{Kbp}$ were inspected for even coverage and manually annotated. Annotation was performed using Integrative services for genomic analysis [31] and the BLASTp algorithm at NCBI to determine the presence of conserved motifs. The majority of genes could not be assigned a function, either because all hits with significant homology were either unannotated or predicted proteins, or because the open reading frame lacked homology to any sequence in the nr (non-redundant protein) database (E-value $>10^{5}$ )

To obtain independent rumen virome contigs the reads from [8] were also assembled using Gossamer [30] with $\mathrm{K}$-mer $=60$.

\section{Identifying integrated phage}

Reads from [15] (microbiome sequence from the same samples as the 12 co-habited viromes) were aligned to each of the 14 largest contigs annotated in Figure 1 using BWA [32]. The proportion of alignments was calculated as $A / B$, where $\mathrm{A}$ is the number of reads that aligned to that contig, and $\mathrm{B}$ is the number of reads that aligned to all 14 contigs. The same procedure was applied to the virome data. Differences between the proportion of reads that aligned from the virome and microbiome were assessed by a paired t-test with two tails. A Bonferroni correction was applied by multiplying each p-value by the number of tests (14). To ensure that the overrepresentation in the microbiome was not due to a single conserved gene, alignments from the microbiome on the two significant contigs were manually checked. Converge of the contigs was relatively even, suggesting that the entire sequence was over represented in the microbiome (compared to expected contamination proportions)

\section{Richness}

Overlapping reads were joined together using FLASH [33]. To estimate the number of species present in each of our samples, we used CatchAll [13], employing the minimo assembler within Circonspect [34] (the parameters changed from default were -u 20 -v 100 -r 1 -1 35 -s 100000) on 100000 joined reads.

\section{Between sample comparison}

To compare the abundance of each contig in each sample the sequence reads were mapped to the assembled contigs using BWA-backtrack [32], with no insertions/deletions permitted. To compare the abundance of KEGG pathways we used 100,000 joined reads per sample and preformed a BLASTx search of the nr (non redundant protein) database of reads. Of the reads, $16.4 \%$ had a hit to the database with extremely relaxed parameters (e-value $<0.02)$. We then used MEGAN [35,36] (default parameters) to extract KEGG assignments form the BLAST output.

\section{Competing interests}

The authors declare no competing interests.

\section{Author's information}

Steve Petrovski is currently at Peter MacCallum Cancer Centre, Melbourne Australia. 


\section{Authors' contributions}

The parent study was run by PM. Samples were collected by PM and ER. Laboratory work was completed by SP. Analysis was performed and the manuscript written by ER. Interpretation of results, critique and final editing of the manuscript was performed by ER, SP, PM and BH. All authors have seen and approved the manuscript.

\section{Acknowledgments}

The authors are very grateful to the Dairy Futures Cooperative Research Centre for funding this work. Funding for this work was also provided by the Department of Environment and Primary Industries Victoria (DEPI), Dairy Australia, Meat and Livestock Australia, and the Australian Government Department of Agriculture, Fisheries and Forestry under its Australia's Farming Future Climate Change Research Program. Thank you also to the staff of DEPI Ellinbank who helped with sampling and the staff of DEPI Bundoora who helped with molecular techniques, Andrew Robinson (La Trobe University) for helping with the CatchAll analysis and Professor Ben Cocks (DEPI) for comments on the manuscript.

\section{Author details}

'Department of Environment and Primary Industries, Bundoora, VIC 3086 Australia. ${ }^{2}$ Dairy Futures Cooperative Research Centre, Bundoora, VIC 3086 Australia. ${ }^{3}$ La Trobe University, Bundoora, VIC 3086, Australia.

Received: 13 May 2013 Accepted: 24 October 2013

Published: 1 November 2013

\section{References}

1. Van Soest PJ: Nutritional ecology of the ruminant. Ithaca, NY: Cornell Univ. Press; 1994

2. Deng W, Xi D, Mao H, Wanapat M: The use of molecular techniques based on ribosomal RNA and DNA for rumen microbial ecosystem studies: a review. Mol Biol Rep 2008, 35(2):265-274.

3. Mann NH: The third age of phage. PLOS Biol 2005, 3:182.

4. Brulc JM, Antonopoulos DA, Berg Miller ME, Wilson MK, Yannarell AC, Dinsdale EA Edwards RE, Frank ED, Emerson JB, Wacklin P, et al: Gene-centric metagenomics of the fiber-adherent bovine rumen microbiome reveals forage specific glycoside hydrolases. Proc Natl Acad Sci U S A 2009, 106(6):1948-1953.

5. Hess M, Sczyrba A, Egan R, Kim T-W, Chokhawala H, Schroth G, Luo S, Clark DS, Chen F, Zhang T, et al: Metagenomic discovery of biomass-degrading genes and genomes from cow rumen. Science 2011, 331(6016):463-467.

6. Ross EM, Moate PJ, Bath CR, Davidson SE, Sawbridge TI, Guthridge KM, Cocks $B G$, Hayes BJ: High throughput whole rumen metagenome profiling using untargeted massively parallel sequencing. BMC Genet 2012, 13:53.

7. Li RW, Connor EE, Li C, Baldwin V, Ransom L, Sparks ME: Characterization of the rumen microbiota of pre-ruminant calves using metagenomic tools. Environ microbiol 2012, 14(1):129-139.

8. Berg Miller ME, Yeoman CJ, Chia N, Tringe SG, Angly FE, Edwards RA, Flint HJ Lamed R, Bayer EA, White BA: Phage-bacteria relationships and CRISPR elements revealed by a metagenomic survey of the rumen microbiome. Environ Microbio/ 2012, 14(1):207-227.

9. Swain RA, Nolan JV, Klieve AV: Natural variability and diurnal fluctuations within the bacteriophage population of the rumen. Appl Environ Microbiol 1996, 62:994-997.

10. Reyes A, Semenkovich NP, Whiteson K, Rohwer F, Gordon Jl: Going viral: next-generation sequencing applied to phage populations in the human gut. Nat Rev Microbiol 2012, 10(9):607-617.

11. Angly FE, Willner D, Prieto-Davó A, Edwards RA, Schmieder R, Vega-Thurber $R$, Antonopoulos DA, Barott K, Cottrell MT, Desnues C, et al: The GAAS Metagenomic Tool and Its Estimations of Viral and Microbial Average Genome Size in Four Major Biomes. PLoS Comput Biol 2009, 5(12):e1000593.

12. Angly $F$, Rodriquez-Brito $B$, Bangor D, McNairnie $P$, Breitbart M, Salamon $P$, Felts B, Nulton J, Mahaffy J, Rohwer F: PHACCS, an online tool for estimating the structure and diversity of uncultured viral communities using metagenomic information. BMC bioinform 2005, 6(1):41.

13. Allen HK, Bunge J, Foster JA, Bayles DO, Stanton TB: Estimation of viral richness from shotgun metagenomes using a frequency count approach. Microbiome 2013, 1:5.

14. Reyes A, Haynes M, Hanson N, Angly FE, Heath AC, Rohwer F, Gordon J: Viruses in the faecal microbiota of monozygotic twins and their mothers. Nature 2010, 466(7304):334-338.
15. Ross EM, Moate PJ, Marett L, Cocks BG, Hayes BJ: Investigating the effect of two methane mitigating diets on the rumen microbiome using massively parallel sequencing. J dairy sci 2013. 96(9):6030-6046.

16. Letarov A, Kulikov E: The bacteriophages in human- and animal bodyassociated microbial communities. J Appl Microbio/ 2009, 107(1):1-13.

17. Coetzee B, Freeborough M-J, Maree HJ, Celton J-M, Rees DJG, Burger JT: Deep sequencing analysis of viruses infecting grapevines: Virome of a vineyard. Virology 2010, 400(2):157-163.

18. Allen HK, Looft T, Bayles DO, Humphrey S, Levine UY, Alt D, Stanton TB: Antibiotics in Feed Induce Prophages in Swine Fecal Microbiomes. mBio 2011, 2:6.

19. Pride DT, Salzman J, Haynes M, Rohwer F, Davis-Long C, White RA III, Loomer P, Armitage GC, Relman DA: Evidence of a robust resident bacteriophage population revealed through analysis of the human salivary virome. ISME Journal 2012, 6(5):915-926.

20. Rodriguez-Brito B, Li L, Wegley L, Furlan M, Angly F, Breitbart M, Buchanan J, Desnues C, Dinsdale E, Edwards R, et al: Viral and microbial community dynamics in four aquatic environments. ISME J 2010, 4(6):739-751.

21. Willner D, Furlan M, Schmieder R, Grasis JA, Pride DT, Relman DA, Angly FE, McDole T, Mariella RP, Rohwer F, et al: Metagenomic detection of phageencoded platelet-binding factors in the human oral cavity. Proc Natl Acad Sci 2011, 108:4547-4553

22. Lamendella R, Santo Domingo JW, Ghosh S, Martinson J, Oerther DB: Comparative fecal metagenomics unveils unique functional capacity of the swine gut. BMC microbiol 2011, 11(1):103.

23. Sedghizadeh PP, Yooseph S, Fadrosh DW, Zeigler-Allen L, Thiagarajan M, Salek H, Farahnik F, Williamson SJ: Metagenomic investigation of microbes and viruses in patients with jaw osteonecrosis associated with bisphosphonate therapy. Oral Surg Oral Med Oral Pathol Oral Radiol 2012, 114(6):764-770.

24. Hackmann TJ, Spain JN: Invited review: Ruminant ecology and evolution: Perspectives useful to ruminant livestock research and production. J dairy sci 2010, 93(4):1320-1334.

25. Patra A: Enteric methane mitigation technologies for ruminant livestock: a synthesis of current research and future directions. Environ Monit Assess 2012, 184(4):1929-1952.

26. Barr JJ, Auro R, Furlan M, Whiteson KL, Erb ML, Pogliano J, Stotland A Wolkowicz R, Cutting AS, Doran KS, et al: Bacteriophage adhering to mucus provide a non-host-derived immunity. Proc Natl Acad Sci 2013, 110:10771-10776.

27. Lu TK, Collins JJ: Engineered bacteriophage targeting gene networks as adjuvants for antibiotic therapy. Proc Natl Acad Sci U S A 2009, 106(12):4629-4634.

28. Moate PJ, Williams SRO, Ribaux BE, Wales WJ: Feeding grape marc to dairy cows suppresses methane emissions, The 5th Australasian Dairy Science Symposium. 2012:454-455

29. Petrovski S, Seviour RJ, Tillett D: Genome sequence and characterization of the Tsukamurella bacteriophage TPA2. App/ Environ Microbiol 2011, 77(4):1389-1398.

30. Conway T, Wazny J, Bromage A, Zobel J, Beresford Smith B: Gossamer-a resource-efficient de novo assembler. Bioinform (Oxford England) 2012, 28(14):1937-1938.

31. Hemmerich C, Buechlein A, Podicheti R, Revanna KV, Dong Q: An Ergatisbased prokaryotic genome annotation web server. Bioinform (Oxford England) 2010, 26(8):1122-1124.

32. Li H, Durbin R: Fast and accurate short read alignment with BurrowsWheeler transform. Bioinformatics (Oxford, England) 2009, 25(14):1754-1760.

33. Magoc T, Salzberg SL: FLASH: Fast Length Adjustment of Short Reads to Improve Genome Assemblies. Bioinform (Oxford England) 2011 27:2957-2963

34. Angly FE, Felts B, Breitbart M, Salamon P, Edwards RA, Carlson C, Chan AM Haynes M, Kelley S, Liu H: The marine viromes of four oceanic regions. PLoS biol 2006, 4(11):e368.

35. Huson DH, Auch AF, Qi J, Schuster SC: MEGAN analysis of metagenomic data. Genome res 2007, 17(3):377-386.

36. Huson DH, Mitra S, Ruscheweyh H-J, Weber N, Schuster SC: Integrative analysis of environmental sequences using MEGAN4. Genome res 2011, 21:1552-1560

doi:10.1186/1471-2180-13-242

Cite this article as: Ross et al:: Metagenomics of rumen bacteriophage from thirteen lactating dairy cattle. BMC Microbiology 2013 13:242. 